Appendix 1. Maternal and Obstetric Comorbidities, ICD-9 Codes, and Minimum Level of Maternal Care

\begin{tabular}{|c|c|c|}
\hline Maternal Comorbidity & ICD-9-CM Code & $\begin{array}{l}\text { Minimum Level } \\
\text { of Care }\end{array}$ \\
\hline Preterm & 644.x & $\mathrm{I}$ \\
\hline Multiple Gestation & V27.2-V27.8, 651.x & I \\
\hline Previous Cesarean Delivery & $654.2 \mathrm{x}$ & I \\
\hline Prior Uterine Surgery & $654.9 x$ & I \\
\hline Gestational Hypertension & $642.3 x$ & I \\
\hline Preeclampsia without Severe & $642.4 x, 642.7 x$ & I \\
\hline \multicolumn{3}{|l|}{ Features } \\
\hline Chronic Hypertension & $\begin{array}{l}\text { 401.x, 402.x, 403.x, 404.x, 405.x, } \\
\text { 642.0x, 642.1x, 642.2x, 642.7x }\end{array}$ & I \\
\hline Gestational Diabetes & $648.8 x$ & I \\
\hline Preterm Multiple Gestation & V27.2-V27.8, 651.x AND 644.x & II \\
\hline Pre-Existing Diabetes Mellitus & 250.x, 648.0x & II \\
\hline Alcohol Abuse & 291.xx, 303.xx, 305.0x & II \\
\hline Drug Abuse & 304.x, 305.2-305.9x, 648.3x & II \\
\hline Asthma & 493.x & II \\
\hline History of DVT or PE & V12.51, V12.55 & II \\
\hline Hypercoagulable State & 289.81, 289.82 & II \\
\hline $\begin{array}{l}\text { Easter SR, Robinson JN, Menard } \\
\text { regionalized maternity care on u } \\
\text { The authors provided this inform } \\
\text { (C)2019 American College of Obs }\end{array}$ & $\begin{array}{l}\text { eanga AA, Xu X, Little SE, et al. Pote } \\
\text { itals. Obstet Gynecol 2019;134. } \\
\text { s a supplement to their article. } \\
\text { ns and Gynecologists. }\end{array}$ & tial effects of \\
\hline
\end{tabular}


Coagulation Disorder

649.3x

Abruption

641.x, 656.0

II

Preterm Chronic Hypertension

401.x, 402.x, 403.x, 404.x, 405.x, II

642.0x, 642.1x, 642.2x, 642.7x

AND 644.x

Preterm Gestational Hypertension

642.3x AND 644.x

II

Preterm Preeclampsia without

642.4x, 642.7x AND 644.x

II

Severe Features

Preeclampsia with Severe Features

$642.5 x, 642.6 x$

II

Placenta Previa

641.0x, 641.1x

II

Previa with Prior Uterine Surgery

641.0x, 641.1x AND 654.9x OR

III

$654.2 x$

Preterm Preeclampsia with Severe

642.5x, 642.6x AND 644.x

III

Features

Chronic Renal Disease

581.x-583.x, 585.x, 587.x, 588.x,

III

$646.2 x$

Cystic Fibrosis

277.0x

III

Epilepsy

649.1, 694.4x

III

HIV

042.x, V08.x

III

Systemic Lupus Erythematosus

710.0x

III

Sickle Cell Disease

$282.4 x, 282.6 x$

III

Hematologic Cancer

200.x - 209.x

III

Easter SR, Robinson JN, Menard MK, Creanga AA, Xu X, Little SE, et al. Potential effects of regionalized maternity care on u.s. hospitals. Obstet Gynecol 2019;134.

The authors provided this information as a supplement to their article.

(C)2019 American College of Obstetricians and Gynecologists. 
Breast Cancer

Maternal Cancer

14x.x, 15x.x, 160.x-165.x, 170.x,

III

171.x, 188.x, 189.x

Gynecologic Cancer

179.x, 180.x, 181.x, 182.x, 183.x,

III

184.x

Congenital Heart Disease

745.0x-747.4x, 648.5x

IV

Chronic Ischemic Heart Disease

412.x-414.x

IV

Cardiac Valvular Disease

394.x-397.x, 424.x

IV

Endocarditis

424.9x, 421.x

IV

Aortic Stenosis

395.0, 395.2, 746.3, 746.81,

IV

747.22

Mitral Stenosis

394.0, 394.2, 746.5

IV

Aortic and Mitral Stenosis

396.0, 396.2, 396.8

IV

Mechanical Heart Valve

V43.3

IV

Hypertrophic Cardiomyopathy

$425.1 x$

IV

Chronic Congestive Heart Failure

428.22, 428.23, 428.32, 428.33,

IV

428.42, 428.43

Marfan Syndrome

759.82

IV

Aortic Aneurysm

441.x

IV

Pulmonary Hypertension

416.0x, 416.8x, 416.9x

IV

Hypoplastic Left Heart Syndrome

746.7

IV

Easter SR, Robinson JN, Menard MK, Creanga AA, Xu X, Little SE, et al. Potential effects of regionalized maternity care on u.s. hospitals. Obstet Gynecol 2019;134.

The authors provided this information as a supplement to their article.

(C)2019 American College of Obstetricians and Gynecologists. 
Single Ventricle (Fontan)

Cirrhosis

Brain Tumor

Neurofibromatosis
745.3

571.5, 571.6

225.0, 225.1, 225.2, 191.x

237.70, 237.71, 237.72, 237.70
IV

IV

IV

IV

Easter SR, Robinson JN, Menard MK, Creanga AA, Xu X, Little SE, et al. Potential effects of regionalized maternity care on u.s. hospitals. Obstet Gynecol 2019;134.

The authors provided this information as a supplement to their article.

(C)2019 American College of Obstetricians and Gynecologists. 
Appendix 2. Hospital Services, SID Procedure Code or AHA Code, and Hospital Level of

Care

\begin{tabular}{llcc}
\hline \multicolumn{1}{c}{ Definition } & SID or AHA Description & $\begin{array}{c}\text { ICD9 Procedure } \\
\text { Code }\end{array}$ & AHA Variable \\
& & & \\
\hline Level II & & $47 . \mathrm{x}$ & \\
\hline General Surgery & Appendectomy Procedure & $45 . \mathrm{x}$ & \\
Services & & & \\
& Bowel Resection Procedure & $512 . \mathrm{x}$ & \\
& Cholecystectomy Procedure & & MSICHOS \\
Intensive Care Unit & Medical/Surgical ICU & & ULTSNHOS \\
Ultrasound & US in Hospital & $887 . \mathrm{x}$ & \\
& US Procedure & & CTSCNHOS \\
CT Scan & CT in Hospital & $870.3,870.4$, & \\
& CT Procedure & $877.1,874.1$, & \\
& & 880.1 &
\end{tabular}

MRI

MRI in Hospital

MRIHOS

MRI Procedure $\quad$ 889.x

\section{Level III}

Cardiology

Cardiac Services Available

CICHOS

Cardiac ICU Beds

CIBD $>1$

Adult Cardiology Available

ACARDHOS

Easter SR, Robinson JN, Menard MK, Creanga AA, Xu X, Little SE, et al. Potential effects of regionalized maternity care on u.s. hospitals. Obstet Gynecol 2019;134.

The authors provided this information as a supplement to their article.

(C)2019 American College of Obstetricians and Gynecologists. 
Neurology

Neurology Services Available

NEROHOS

Nephrology

Hemodialysis Available

HEMOHOS

\section{Level IV}

\begin{tabular}{llc}
\hline Transplant Services & Heart Transplant & HARTHOS \\
& Kidney Transplant & KDNYHOS \\
& Liver Transplant & LIVRHOS \\
& Lung Transplant & LUNGHOS \\
Cardiac Surgery & Cardiac Surgery & ASTCHOS \\
& Valve Procedures & \\
Neurosurgery & Neurosurgical Procedures & \\
${ }^{*}$ Indicates all codes or services must be present for hospital with given AHA ID to meet \\
definition of available services.
\end{tabular}

Easter SR, Robinson JN, Menard MK, Creanga AA, Xu X, Little SE, et al. Potential effects of regionalized maternity care on u.s. hospitals. Obstet Gynecol 2019;134.

The authors provided this information as a supplement to their article.

(C)2019 American College of Obstetricians and Gynecologists. 
Appendix 3. Hospital Delivery Level and Receipt of Inappropriate Level of Care

According to State of Delivery

\begin{tabular}{|c|c|c|c|c|c|}
\hline $\begin{array}{l}\text { State of } \\
\text { Hospital }\end{array}$ & $\begin{array}{c}\text { Level I } \\
(\mathrm{n}=103,987)\end{array}$ & $\begin{array}{c}\text { Level II } \\
(\mathrm{n}=\mathbf{2 8 5 , 6 8 5 )}\end{array}$ & $\begin{array}{c}\text { Level III } \\
(\mathrm{n}=304,764)\end{array}$ & $\begin{array}{c}\text { Level IV } \\
(\mathrm{n}=151,109)\end{array}$ & $\begin{array}{c}\text { Inappropriately } \\
\text { Low Level }\end{array}$ \\
\hline Florida & $\begin{array}{l}52,369 \\
(24.77)\end{array}$ & $\begin{array}{l}56,504 \\
(26.72)\end{array}$ & $\begin{array}{l}68,118 \\
(32.21)\end{array}$ & $\begin{array}{l}34,459 \\
(16.30)\end{array}$ & $8,910(4.21)$ \\
\hline Massachusetts & $\begin{array}{l}8,788 \\
(12.60)\end{array}$ & $\begin{array}{l}24,026 \\
(34.46)\end{array}$ & $\begin{array}{l}13,993 \\
(20.07)\end{array}$ & $\begin{array}{l}22,917 \\
(32.87)\end{array}$ & $1,558(2.23)$ \\
\hline New Jersey & $\begin{array}{l}6,866 \\
(6.95)\end{array}$ & $\begin{array}{l}30,274 \\
(30.65)\end{array}$ & $\begin{array}{l}41,001 \\
(41.50)\end{array}$ & $\begin{array}{l}20,645 \\
(20.90)\end{array}$ & $1,515(1.53)$ \\
\hline New York & $\begin{array}{l}20,080 \\
(8.74)\end{array}$ & $\begin{array}{l}54,873 \\
(23.89)\end{array}$ & $\begin{array}{l}108,259 \\
(47.12)\end{array}$ & $\begin{array}{l}46,525 \\
(20.25)\end{array}$ & 4,238 (1.84) \\
\hline North Carolina & $\begin{array}{l}4,573 \\
(4.02)\end{array}$ & $\begin{array}{l}44,185 \\
(38.87)\end{array}$ & $\begin{array}{l}48,120 \\
(42.33)\end{array}$ & $\begin{array}{l}16,794 \\
(14.77)\end{array}$ & $1,431(1.26)$ \\
\hline Oregon & $\begin{array}{l}2,240 \\
(5.26)\end{array}$ & $\begin{array}{l}25,042 \\
(58.84)\end{array}$ & $\begin{array}{l}10,404 \\
(24.45)\end{array}$ & $\begin{array}{r}4,870 \\
(11.44)\end{array}$ & $766(1.80)$ \\
\hline Washington & $\begin{array}{l}9,071 \\
(11.39)\end{array}$ & $\begin{array}{l}50,781 \\
(63.78)\end{array}$ & $\begin{array}{l}14,869 \\
(18.67)\end{array}$ & $\begin{array}{l}4,899 \\
(6.15)\end{array}$ & $1,978(2.48)$ \\
\hline
\end{tabular}

Easter SR, Robinson JN, Menard MK, Creanga AA, Xu X, Little SE, et al. Potential effects of regionalized maternity care on u.s. hospitals. Obstet Gynecol 2019;134.

The authors provided this information as a supplement to their article.

(C)2019 American College of Obstetricians and Gynecologists. 